\title{
DOI 10.3868/s110-004-015-0034-5
}

How Chinese learn mathematics: Perspectives from insiders. Lianghuo Fan, Ngai-Ying Wong, Jinfa Cai, \& Shiqi Li (Eds.). Singapore: World Scientific Publishing, 2004. 574 pp., (paperback), 52\$, ISBN: 981-270-414-0.

This is the first edited book which focuses on understanding the Chinese way of learning mathematics from the perspectives of insiders, as its editors and authors mostly grew up and had their formal education in various regions of China. Many also received research training in the West and some are still working in different parts of the world. In addition, many chapters present their arguments and data directly from a comparative perspective. Therefore, the book is not simply the views of insiders but views in a comparative perspective, which adds real value for understanding the Chinese way of learning mathematics.

While focusing on the Chinese way of learning mathematics, the book can be seen as part of broader efforts to capture the constant and changing characteristics of Chinese learners and the contexts shaping them. These efforts are challenging many assumptions about learning and learners in the Western literature. Thus, the book is valuable for those who are interested in mathematics learning and teaching and those who are interested in the relationship between learning, teaching, and the contexts of teaching and learning in general.

Since ethnic Chinese students in various places have been the top mathematics performers in various international comparisons, the "Chinese" way of learning mathematics can be naturally assumed to contribute to such performances. Characterizing and verifying this way of learning can thus not only help explain why Chinese students perform well, but also allows a unique opportunity to verify, modify, and advance various assumptions about mathematics learning and teaching. However, the conceptual characterization and empirical verification of such learning is difficult as the curriculum and teaching contexts and the cultural and social environments that shape mathematics performance among different groups of Chinese students are substantially varied. These variations are supposed to lead students to different instead of similar levels performance if we follow assumptions in the Western literature, such as that teaching quality is the major influence on student learning, that curriculum standards and accountability assessments shape teaching and its transformation, and that social and cultural 
environments shape the influences of school and teaching on student learning.

The book takes up this valuable yet challenging task and develops a set of new understandings about the Chinese way of learning mathematics and its influential contexts. First, it captures two features of Chinese mathematics learners. As the top mathematics performers in the world, Chinese students are strong in using computation, applying symbolic ideas, conventional strategies, and multiple solutions to solving simple mathematical problems but not as strong in data analysis, graphs, open-ended problem solving, risk taking and using concrete drawing in solving open-ended problems. Chinese students also hold certain beliefs about learning rooted in traditional Chinese culture, which stress the dialectic relationships between ability and effort, experiences of success and failure, extrinsic and intrinsic motivation, and individual perfection and social contribution goals in shaping their learning, instead of seeing these pairs of beliefs as dichotomous in the way their U.S. counterparts do.

Second, it offers a few characteristics of the Chinese mathematics curriculum. Ingrained in its historical tradition, the Chinese mathematics curriculum emphasizes students' mastery of basic concepts and their relationships and students' efficient and flexible application of basic skills in solving problems. Part of the curriculum is the set of centralized textbooks developed following the national curriculum standards, which are the major source for teachers in making mathematics teaching decisions and for students in doing mathematics exercises in school and at home. Another part is the centralized teaching manuals, which put pressure on teachers, support them in understanding the textbook content and offer them specific pedagogical suggestions for their instruction. Under the influence of globalization, this curriculum is changing with a de-emphasis on symbolic computation, flexible reasoning, and topic coverage and an increase in student investigations and understanding about the context of investigations.

Third, it identifies several features of Chinese mathematics teaching. Chinese classrooms under the dominant control of teachers show a strong coherence in helping students understand a concept through connections among various concepts and applying the concept using varied approaches through sequential classroom activities. Such teaching helps students form varied cognitive structures about a concept and engages them more actively in constructing and analyzing mathematics ideas. Chinese teachers' beliefs about the roles of both conceptual and procedural knowledge in developing mathematics instruction and 
student mathematics thinking, shape such teaching in important ways. However, such teaching is not practiced in the same ways in different Chinese regions nor with equal quality. Teachers in Hong Kong tend to use physical manipulation in concept exploration and visual presentations in concept practices while those in Shanghai prefer to use numerical operations in concept exploration and symbolic presentations in practice. Although similar in terms of teacher-dominant communications, ultimate dependence on textbook examples, and the primary use of teaching time for practice, teachers in Chinese urban schools offer more inspiring questions, diverse learning activities, and more frequently accommodate students' difficulties than those in rural schools. Such teaching is being transformed toward an approach featuring more student investigation and experimentation, which is not always beneficial for students' accurate understanding of mathematics concepts.

Finally, it highlights two non-school influences on the Chinese way of learning mathematics. After-school mathematics programs are a popular extension of Chinese students' school mathematics learning, which help students improve computation skills, complete school homework, and boost their confidence in mathematics learning while compromising their conceptual understanding and flexible use of mathematics concepts. The traditional heritage, such as Confucian cultural values, could script the Chinese way of mathematics teaching and learning. The scripted nature of learning and teaching makes it difficult for teachers to borrow teaching and learning practices that are effective somewhere else and implement them in their own classrooms.

This book leads me directly to several unresolved questions. Why do Chinese students' beliefs that reflect the traditional Chinese cultural values fail to influence all the areas of mathematics learning positively among various Chinese groups? What are the empirical relationships between the Chinese mathematics curriculum and teaching approach, and the Chinese way of mathematics learning as documented? To what extent will the reform of the Chinese mathematics curriculum and its teaching lead to change in the Chinese way of mathematics learning as expected? How do the variations within Chinese cultural traditions and the social and economic contexts shape mathematics teaching and learning in various places differently? The progress made and the questions left by this book will surely inspire further conceptualization and exploration into the Chinese way 
of learning mathematics.

\author{
Jian WANG \\ Texas Tech University \\ E-mail: jian.wang@ttu.edu
}

DOI 10.3868/s110-004-015-0035-2

Vocational education of female entrepreneurs in China: A multitheoretical and multidimensional analysis of successful businesswomen's everyday lives. Mary Ann Maslak. Abingdon, England: Routledge, 2015. 110 pp., (hardcover), 145\$, ISBN: 978-0-415-73742-5.

This book is about vocational education, but also about possibilities of non-/informal education; it is about China, but also about a specific ethnic minority group (the Dongxiang), which is, among other things, characterized by Islamic faith; it is about entrepreneurship, and more specifically about successful female business women; it is about these women's everyday lives; and, if that was not enough, the author calls her analysis also "multitheoretical." Any reader who might now be expecting a weighty tome will be surprised to notice that Mary Ann Maslak's book contains a running text of a little more than 80 pages. As might have been foreknown, this brevity compromises both scope and depth of an analysis that otherwise could have been insightful and multi-faceted.

The book's topic is wisely chosen: How can we think in educational terms about people who usually do not belong to the target group of vocational education (in this case, illiterate women residing in rather remote, impoverished regions)? And may successful cases among these groups be able to point us to new ways of experimenting with (vocational) education and training on the ground? Maslak sets out to explore these issues by investigating the examples of four comparatively successful business women in a small town in the southern part of Gansu province, Northwest China, whom she met first in 2005 and then re-visited almost ten years later. In her final chapters, Maslak argues that we 